\title{
Rushan earthquake swarm in eastern China and its indications of fluid-triggered rupture
}

\author{
Jian-Chang Zheng $\cdot$ Dong-Mei Li $\cdot$ Cui-Qin Li $\cdot$ Peng Wang • \\ Chang-Peng Xu
}

Received: 24 July 2017/ Accepted: 31 October 2017/Published online: 12 December 2017

(c) The Author(s) 2017. This article is an open access publication

\begin{abstract}
An extraordinary earthquake swarm occurred at Rushan on the Jiaodong Peninsula from October 1, 2013, onwards, and more than 12,000 aftershocks had been detected by December 31, 2015. All the activities of the whole swarm were recorded at the nearest station, RSH, which is located about $12 \mathrm{~km}$ from the epicenter. We examine the statistical characteristics of the Rushan swarm in this paper using RSH station data to assess the arrival time difference, $t_{\mathrm{S}-\mathrm{P}}$, of $\mathrm{Pg}$ and $\mathrm{Sg}$ phases. A temporary network comprising 18 seismometers was set up on May 6 , 2014 , within the area of the epicenter; based on the data from this network and use of the double difference method, we determine precise hypocenter locations. As the distribution of relocated sources reveals migration of seismic activity, we applied the mean-shift cluster method to perform clustering analysis on relocated catalogs. The results of this study show that there were at least 16 clusters of seismic activities between May 6, 2014, and June 30, 2014, and that each was characterized by a hypocenter spreading process. We estimated the hydraulic diffusivity, $D$, of each cluster using envelope curve fitting; the results show that $D$ values range between 1.2 and $3.5 \mathrm{~m}^{2} / \mathrm{d}$ and that approximate values for clusters on the edge of the source area are lower than those within the central area. We utilize an epidemic-type aftershock sequence (ETAS) model to separate external triggered events from self-excited aftershocks within the Rushan swarm. The estimated
\end{abstract}

\section{J.-C. Zheng ( $\square)$}

Institute of Seismology, Chung Cheng University, Chiayi,

Taiwan, China

e-mail: zjcmail@yeah.net

J.-C. Zheng · D.-M. Li · C.-Q. Li · P. Wang · C.-P. Xu

Shandong Earthquake Agency, China Earthquake

Administration, Ji'nan, China parameters for this model suggest that $\alpha=1.156$, equivalent to sequences induced by fluid-injection, and that the forcing rate $(\mu)$ implies just 0.15 events per day. These estimates indicate that around $3 \%$ of the events within the swarm were externally triggered. The fact that variation in $\mu$ is synchronous with swarm activity implies that pulses in fluid pressure likely drove this series of earthquakes.

Keywords Earthquake swarm · Fluid triggered · Cluster analysis · ETAS model

\section{Introduction}

Jiaodong Peninsula is part of the Sulu Orogen and separates the Bohai and Yellow seas in eastern North China. The well-known Tancheng-Lujiang fault zone, which cuts through the Moho discontinuity and spreads out across almost the whole eastern part of Chinese mainland, lies to the west of this peninsula, while the Penglai-Weihai fault, also characterized by a high level of activity, crosses the northern side. The Penglai-Weihai fault is part of the Yanshan-Bohai seismic zone; the devastating $1976 M_{\mathrm{S}} 8.0$ Tangshan earthquake occurred within this seismic zone. To the south, the Qianliyan fault strikes to the northeast, while a series of smaller faults that share this orientation spread out over the Jiaodong Peninsula and control the dominant seismic activity in the region (Fig. 1; Zhang et al. 2006).

Jiaodong Peninsula is characterized by relatively low earthquake activity. Modern earthquake catalog published by CENC (China Earthquake Networks Center) shows that an average of just 2.33 events with $M_{\mathrm{L}} \geq 3.0$ have taken place each year in this region since 1970 and that the largest recorded event before 2013 was an earthquake with $M_{\mathrm{L}}$ 4.5. Records of historical earthquakes indicate that just 


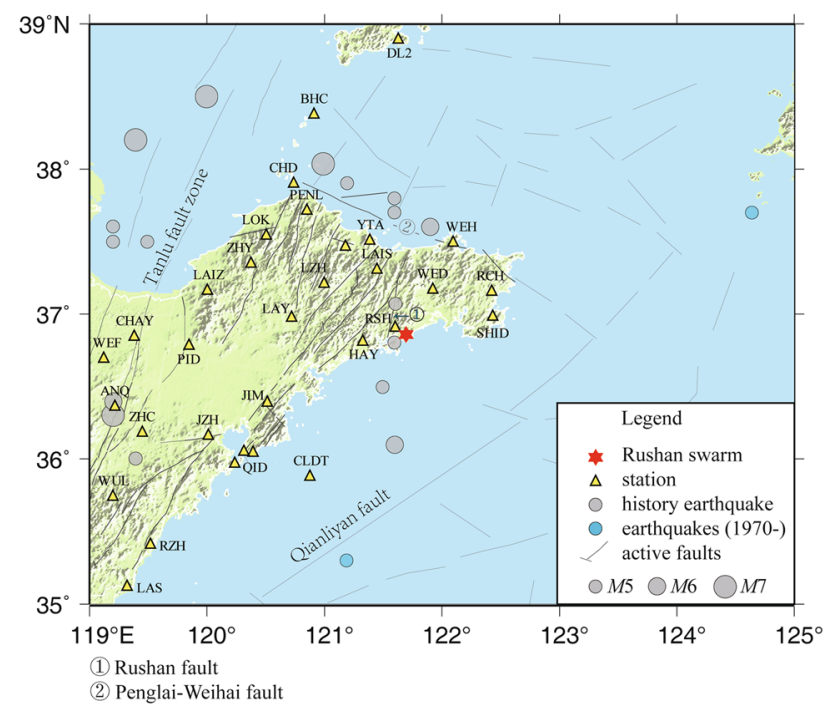

Fig. 1 Map to show the Rushan earthquake swarm (red hexagram) and the regional seismic network (yellow triangles) on the Jiaodong Peninsula. Gray circles mark historical earthquakes $(M \geq 5)$, while light blue circles denote the two modern events occurred in this region since 1970

two medium-strong events (M5.5) have taken place in this region: one in 1046 A.D. and the other in 1939 (Fig. 1). At the same time, however, this region is also well known for the episodic occurrence of earthquake clusters that comprise lots of small magnitude events (Liu et al. 2007; Wang and Zheng 2014).

On October 1, 2013, an earthquake swarm initiated at a location about $15 \mathrm{~km}$ to the southeast of the city of Rushan, on the southern edge of Jiaodong Peninsula. This swarm persisted until 2016, and more than 12,000 aftershocks had been detected by December 31, 2015 (Fig. 2a). The biggest event occurred on May 22, 2015, and had a surface-wave magnitude $\left(M_{\mathrm{S}}\right)$ of 4.6. Although earthquake sequences comprising small-to-medium-sized events are common on the Jiaodong Peninsula (more than 50 have been recorded since 1970), their durations are usually short, encompassing several days up to no more than a month (e.g., the Laoshan swarm in 2003; Zheng et al. 2006). This means that the Rushan earthquake swarm is rather unique for the eastern Chinese mainland, irrespective of its duration or active frequency.

A number of seismological problems are posed by the Rushan earthquake swarm. In the first place, as active faults appear to be absent from the surface of the epicentral area, and even small branch faults have not been identified (Qu et al. 2015; Zheng et al. 2015b), it remains unclear why such a high frequency swarm took place in the area. Secondly, the Rushan event is reminiscent of the wellknown Matsushiro earthquake swarm which was also characterized by high frequency and long duration
(Kishimoto et al. 1967). This latter swarm is thought to be a typical fluid-triggered event by $\mathrm{CO}_{2}$-rich water caused by the intrusion of magma (Stuart and Johnston 1975; Cappa et al. 2009). It remains unclear whether, or not, the Rushan earthquake swarm was also triggered by fluid, while the ultimate origin of this series of events also remains unknown.

In order to address these issues, we initially relocated the Rushan earthquake swarm using data from a temporary network that was deployed in the area of the epicenter. We then adapted the mean-shift cluster method to analyze the spatiotemporal rupture features of this swarm. Finally, we applied an epidemic-type aftershock sequence (ETAS) model in order to differentiate externally triggered events from self-excited aftershocks within the Rushan earthquake swarm.

\section{Data}

The Rushan earthquake swarm occurred on the southern seaward side of the Jiaodong Peninsula and was recorded by 14 stations within the Shandong local earthquake network, all located $150 \mathrm{~km}$ or less from the epicenter. However, because of local terrain limitations, all these stations were either to the north or the west of the swarm, creating an observational gap of more than 120 degrees (Fig. 1). The station nearest to the earthquake swarm was $\mathrm{RSH}$, approximately $15 \mathrm{~km}$ from the epicenter; this station recorded numerous microevents, including some with negative magnitudes (Most microearthquakes with $M_{\mathrm{L}} \leq 0.5$ are only recorded by $\mathrm{RSH}$ station; these events therefore are not located; but still assembled in the catalog for completeness). However, in the aftermath of one event on January 7, $2014\left(M_{\mathrm{L}} 4.6\right)$, it became clear that Rushan earthquake swarm was not comparable to previous events; from this point on, a temporary network of 18 seismometers was deployed (Fig. 4), formally initiated on May 6, 2014.

\subsection{Seismological characteristics}

A catalog of more than 12,000 events are characterized by $M_{\mathrm{C}}=0.5$ (Fig. 2b); thus, the $b$ value of the Rushan earthquake swarm was less than 0.9 (i.e., 0.8667; Fig. 2b). We utilized a robust regression approach to estimate the $b$ value in order to eliminate the influence of outlier points (Holland and Welsch 1977; Yang and Qu 1999) and confirmed the performance of the calculated inter-event time distribution as in previous work (e.g., Hainzl 2004). However, rather than using a traditional power law model for the probability distribution, we generated a histogram of inter-event times and fitted this to a lognormal 

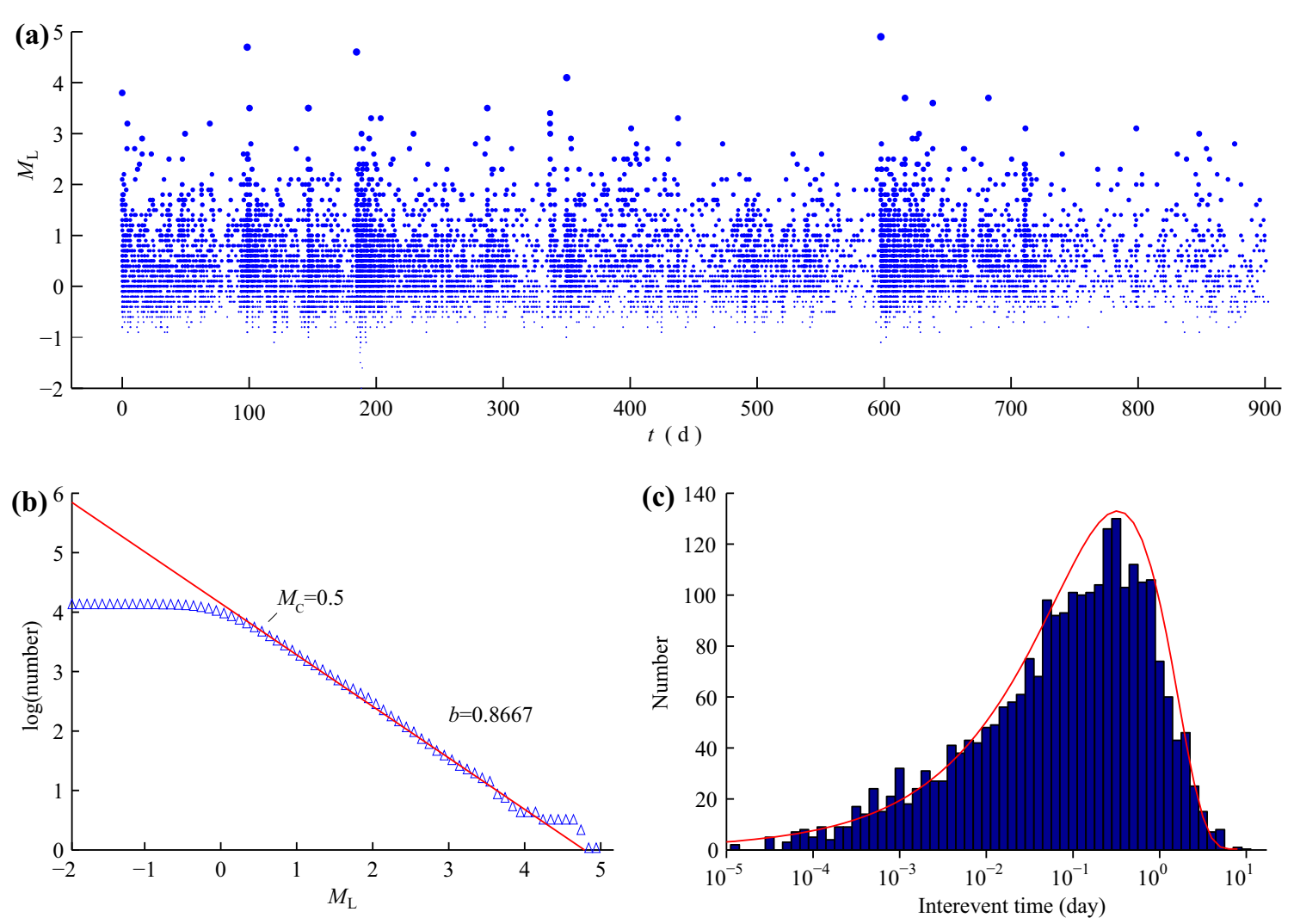

Fig. 2 Statistical characteristics of the Rushan earthquake swarm: a $M_{\mathrm{L}}$ as a function of time recorded from October 1, 2013; b results of Gutenberg-Richter law fitting; $\mathbf{c}$ histogram to show inter-event time (the red solid line denotes the fitted lognormal distribution)

distribution (Fig. 2c). This lognormal distribution can also be considered characteristic of a fractal process; the data presented in Fig. 2c show that the fit is relatively good.

\subsection{Data from the RSH station}

As a temporary seismic network within the area of the epicenter was set up on May 6, 2014, and because the RSH station recorded all swarm activities, we examined differences in the arrival times of $\mathrm{Pg}$ and $\mathrm{Sg}$ phases recorded at the latter. Although small events cannot be located by just a single station, it is nevertheless possible to extract some meaningful information from variation in $t_{\mathrm{S}-\mathrm{P}}$, as these are first-hand data and thus less contaminated by errors than some other phases.

The data show that $t_{\mathrm{S}-\mathrm{P}}$ of almost all the aftershocks falls in the interval $[1.2,1.8]$ second time (Fig. 3). However, prior to April 2014, it is clear that $t_{\mathrm{S}-\mathrm{P}}$ values recorded at the RSH station simultaneously increased and decreased following initiation of the Rushan earthquake swarm. Indeed, two remarkable events (both $M_{\mathrm{L}} \geq 4$ ) were recorded in the early stages of the swarm: one $\left(M_{\mathrm{L}} 4.7\right)$ on January 7, 2014, and the other $\left(M_{\mathrm{L}} 4.6\right)$ on April 4, 2014. The first of these events can be located toward the bottom edge of the $t_{\mathrm{S}-\mathrm{P}}$ plot, while the second occurs toward the top edge, perhaps implying that they can be associated with the source diffusion process of the Rushan earthquake swarm. Although we were unable to determine the direction of aftershock migration, the spatial spreading of swarm activity is obvious and similar to the manner in which epicenters spread within fluid-triggered earthquake swarms (Hainzl 2004; Hainzl and Ogata 2005; Bourouis and Cornet 2009; Hainzl et al. 2012; Shelly et al. 2013a, b). It is noteworthy that this spreading tendency disappeared almost completely after April 2014.

A further point of interest is that an increasingly vacant area formed around a mean value of $t_{\mathrm{S}-\mathrm{P}}$ between May 2014 and April 2015, marked by a dashed magenta line in Fig. 3. Throughout this period, aftershocks took place both proximate and distant to this region, while events $\left(M_{\mathrm{L}} \geq 2\right)$ within the central area were very rare. The largest recorded such event $\left(M_{\mathrm{S}} 4.6\right.$ on May 22, 2015) took place at the end of this vacant area; although this phenomenon is reminiscent of 'seismic quiescence,' noted by Hauksson et al. (2013) in a discussion of the 2012 Brawley earthquake swarm in Southern California, it evolved more rapidly in this case. Thus, another interpretation might be that the blank area corresponds with a barrier encountered within the source area that was broken leading to the mainshock. Actually this gap is an interesting phenomenon, as pointed 


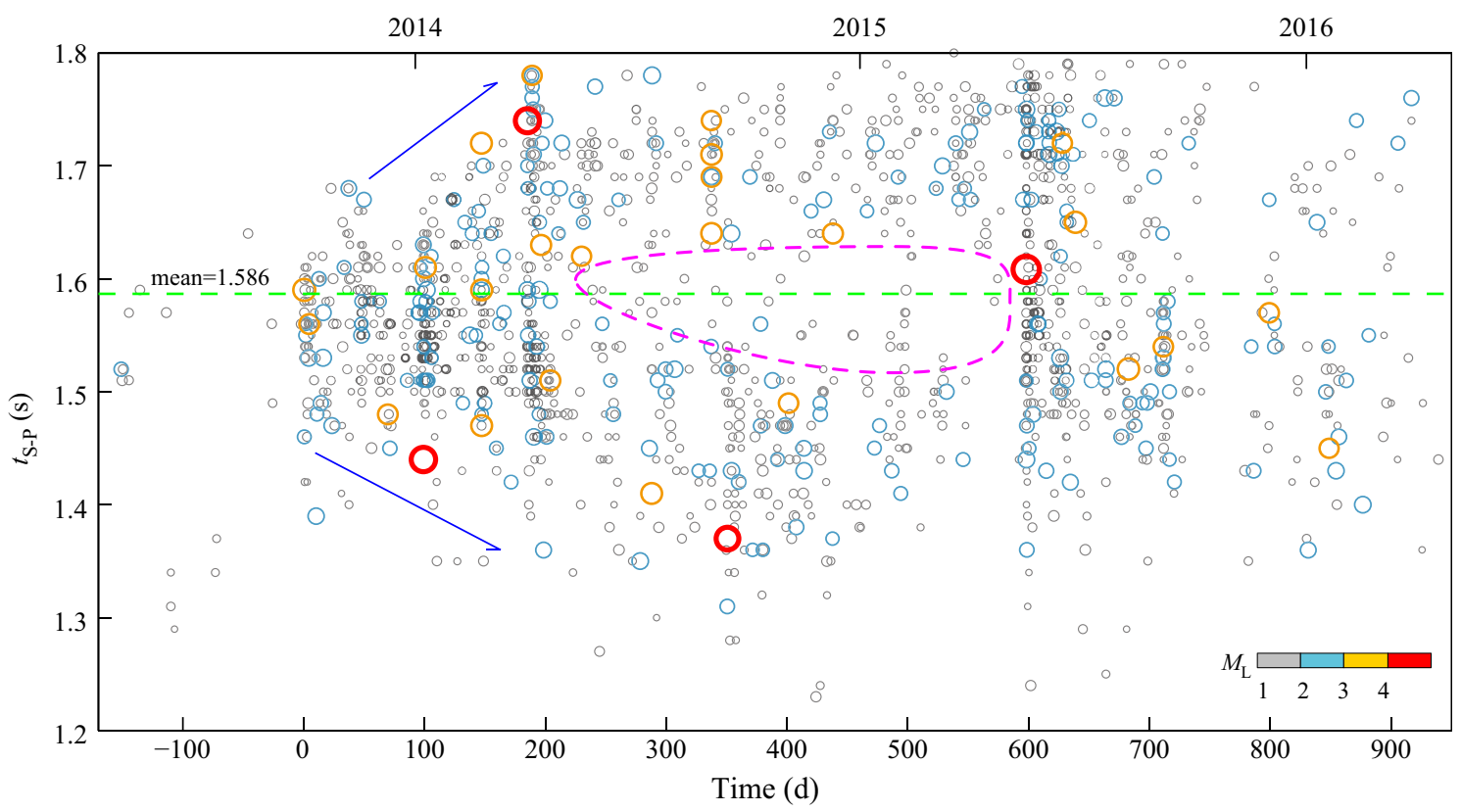

Fig. 3 Data to show arrival time differences in Pg and Sg phases at the RSH station, the dashed green line shows its mean value. Aftershocks were rare within the area outlined by dashed magenta lines, while blue solid arrows mark swarm spreading directions

out in Wei et al. (2013, 2015); this could be caused by the rupture asperity of the big events in the sequence.

\subsection{Relocation based on temporary network data}

As noted above, 18 temporary seismometers were deployed around the edges of the Rushan earthquake swarm (Fig. 4). Although not perfect, the distribution coverage of these seismometers onto swarm activity was acceptable; thus, these instruments, in concert with adjacent regional stations, comprised a temporary network which was used to more comprehensively monitor swarm activity. We have previously compared location results from regional networks with those of temporary ones (Zheng et al. 2015b) and have demonstrated that catalogs based on the former are often unreliable because precise observations are lacking and station coverage can be poor.

We generated a relocated swarm catalog by utilizing temporary network data and the hypoDD method (Waldhauser and Ellsworth 2000, Fig. 4). Thus, using the RSH station as the origin, we established N-E-D coordinates to display these relocated results (Fig. 5). The relocation results show the swarm are clustering between 5 and $8 \mathrm{~km}$ in depth; and the Rushan earthquake swarm strikes in a WNW direction (Fig. 5a), in agreement with the focal mechanism solution for the January 7, 2014, event (i.e., $M_{\mathrm{L}} 4.3$, strike $=298.5^{\circ}$, dip $=64.3^{\circ}$, rake $=0.3^{\circ}$; Zheng et al. 2015a). In this case, the aftershocks were restricted to within a small region $3 \mathrm{~km} \times 3 \mathrm{~km} \times 1 \mathrm{~km}$ in dimensions (Fig. 5a, c, d); this magnitude seismogenic volume is

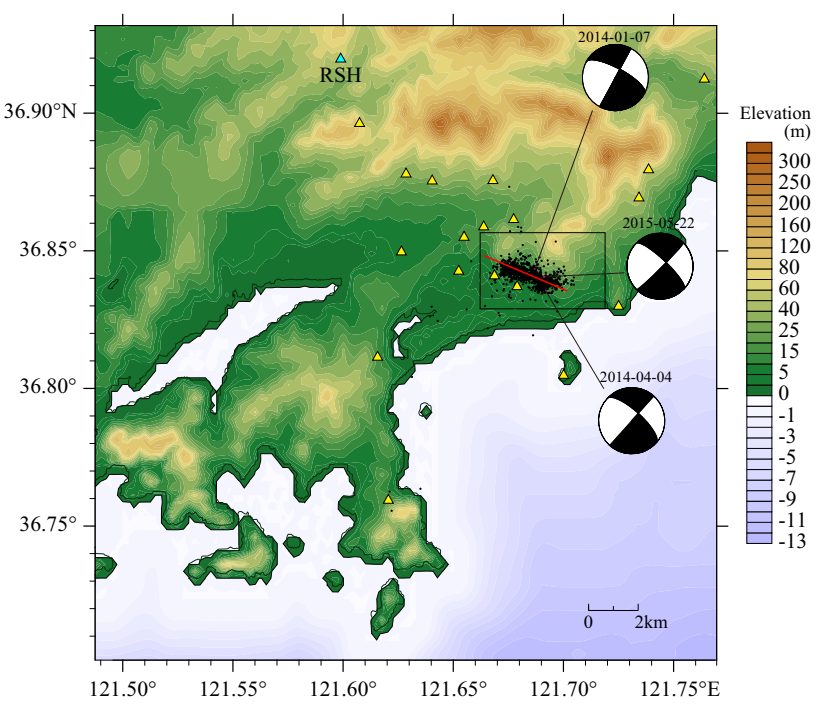

Fig. 4 Distribution of temporary seismometers (yellow triangles) and relocated epicenters (black dots). Focal mechanisms of 3 moderate size events also are shown in the map. The RSH permanent station (blue triangle) belongs to the Shandong regional network. Black solid lines on this figure mark local faults; of these, the Rushan fault (strike between $10^{\circ}$ and $15^{\circ}$ ) is dominant, although two other historical earthquakes (M5.5) are also known to have occurred (see Fig. 1). The dip direction of the Rushan fault is ESE, while its dip angle ranges between $75^{\circ}$ and $85^{\circ}$

similar to that estimated for earthquake swarms in Vogtland and Western Bohemia (Grünthal et al. 1990).

We selected about 2 months of data from our relocated catalog for further analysis in order to examine whether or not the Rushan earthquake swarm was triggered by fluids. 
The relocated catalog starts long after initiation of the earthquake swarm, and there is no evidence for spatial spreading in the distance-time plot (Fig. 5b). However, detailed examination of Fig. $5 c$ reveals that the focus of the swarm is distributed along the fault plane in a particular order (Fig. 5e). Data recorded from May 6, 2014, onwards show that from the first day through to the tenth day, aftershocks were located together within an area between 6.5 and $7.5 \mathrm{~km}$ in depth and between 10 and $11 \mathrm{~km}$ horizontally with respect to the RSH station. In contrast, between the 15th day and the 25th day of the swarm, aftershocks were concentrated below $7.5 \mathrm{~km}$, while between the 30th day and the 40th day they were grouped toward the left part of the fault plane. Finally, after 50 days, swarm aftershocks were focused in two area: one toward the top of the fault plane and the other toward the bottom (Fig. 5e). Data show that the aftershocks not only clustered in stages, also migrated in order and that their hypocenters were distributed as irregular forked branches. All these active phenomena are consistent with the process of crack propagation; comparing our data with seismic activity characteristic of other fluid intrusion-triggered earthquake swarms (e.g., Fig. 8 in Hainzl and Fischer 2002; Figs. 2 and 3 in Hainzl et al. 2012; and studies in Jenatton et al. 2007; Bourouis and Cornet 2009; Shelly et al. 2013a, b) implies that the Rushan example was also likely to have been triggered in this way.

\section{The fluid-triggering hypothesis}

As discussion above, the activity of the Rushan swarm is similar with other fluid-triggered events. Stated by many researchers, variations in pore pressure along a fault plane due to fluid intrusion from a high-pressure source can be described by the diffusion equation as follows:

$\frac{\delta}{\delta t} P=D \frac{\delta^{2}}{\delta x^{2}} P$.

where $D$ is the hydraulic diffusivity, $P$ is pore pressure, and $t$ is the time since the first contact of the pore pressure source with the host rock (Shapiro et al. 1997; Yamashita 1997).

In this case, however, because available observations from the initial stages of the earthquake swarm are limited, no explicit indications for diffusion were noted. In addition, and as discussed above, a precise location catalog is only available subsequent to May 6, 2014, while the distance-time diagram generated from relocations reveals no indications of hypocenter spreading (Fig. 5b). Nevertheless, as also discussed above (see Sect. 2.3), we are able to hypothesize that aftershocks are distributed as irregular forked branches and that they migrated in clusters. These observations suggest that it would be applicable to perform a fluid-triggering analysis for individual clusters over shorter time periods.

\subsection{Cluster analysis}

Cluster analysis is a useful tool in seismology and has been widely applied to locate earthquakes (Frohlich and Davis 1990; Davis and Frohlich 1991; Dzwinel et al. 2005; Shearer et al. 2005; Weatherill and Burton 2009; Rehman et al. 2014), split shear-waves (Teanby et al. 2004) and estimate inversion error (Zheng et al. 2015a).

Godano et al. (2013) demonstrated the presence of multiple earthquake clusters in research on an Italian swarm, while Lindenfeld et al. (2012) recorded several similar clusters in a restricted area while researching fluidtriggered earthquake swarms in the East African Rift. However, while cluster analyses traditionally tended to be density-based, incorporating just spatial coordinates, Zaliapin et al. (2008) introduced a statistical method which defined a unified distance between earthquakes in the timespace-energy domain (Zheng et al. 2014); this approach was subsequently shown to be robust and effective via research in Southern California (Zaliapin and Ben-Zion 2011, 2013a, b).

Building on this earlier work, we applied both temporal and spatial cluster analyses to the data from the Rushan earthquake swarm. We adopted a mean-shift criterion to perform a cluster analysis on relocated catalogs generated for the period between May 6, 2014, and June 30, 2014. Although the mean-shift approach is also density-based (Cheng 1995; Comaniciu and Meer 2002), similar to the $K$ means method applied by many researchers, one advantage is that this technique can be used to detect arbitrary-shaped clusters via an iterative procedure and density estimation.

Results revealed the presence of 16 clusters containing at least 20 events (Fig. 6). Although some isolated events were discarded based on spatiotemporal criteria, the total number retained within our clusters encompassed more than $90 \%$ of the relocated catalog. Clusters were labeled with different colors and arranged from the time of origin of the first event in each case (Fig. 6). For events in same cluster, we suppose they are cracks all outspread from a same origin because they have closer time-space distance. Then, we can estimate the hydraulic diffusivity from these events; for detailed analysis see the next section.

In order to extract information on the processes underlying the Rushan earthquake swarm, we further examined the spatial evolution of clusters over time. The data reveal that cluster 1 was active on the left-central side on the fault plane, while clusters 2-4 moved to the right-bottom side (Fig. 6). Similarly, the aftershocks of clusters 5-9 and cluster 11 spread upwards, cluster 10 and clusters 14 and 
(a)

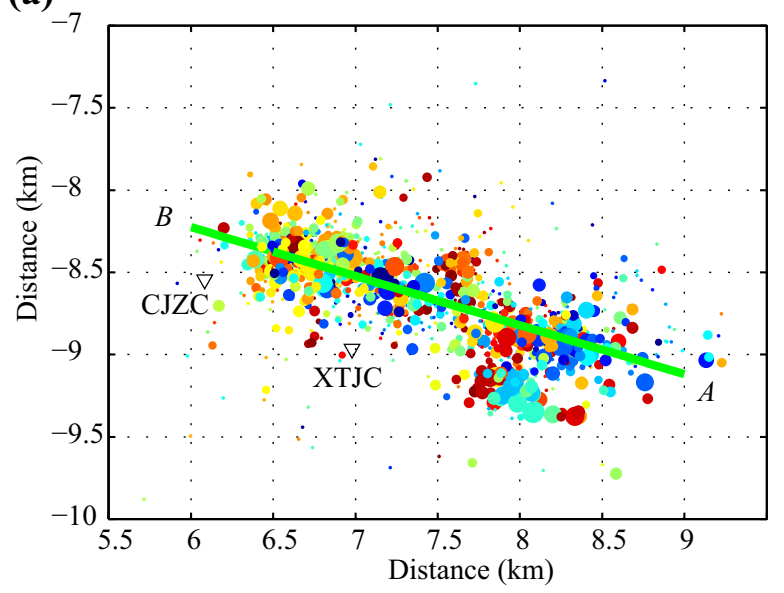

(c)

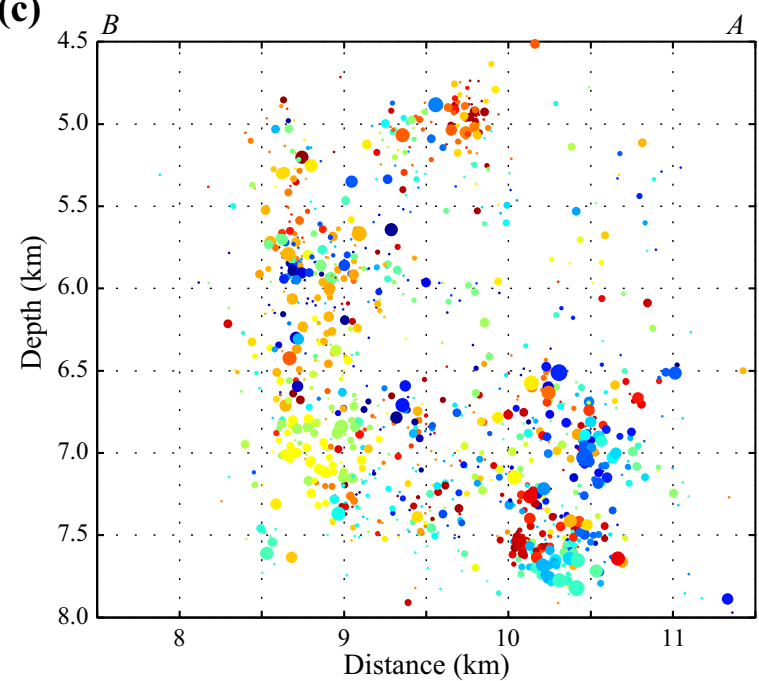

(b)

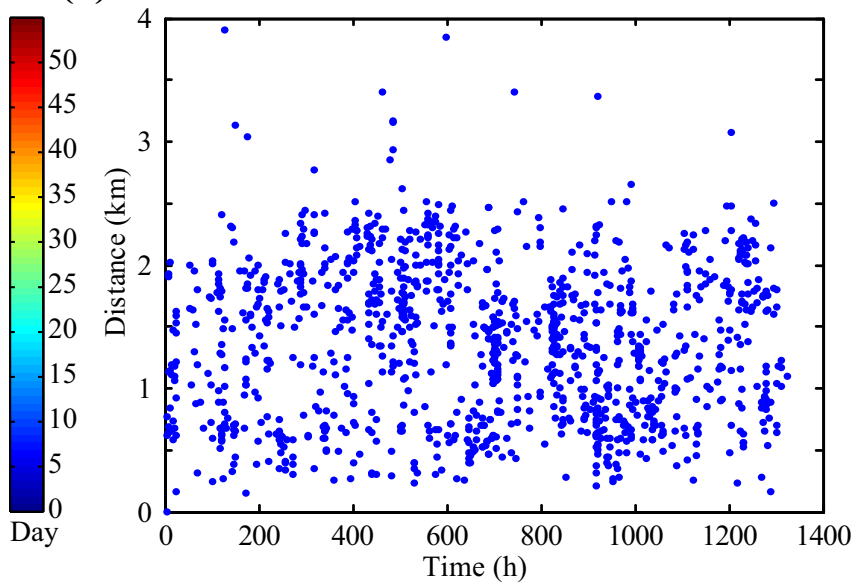

(d)

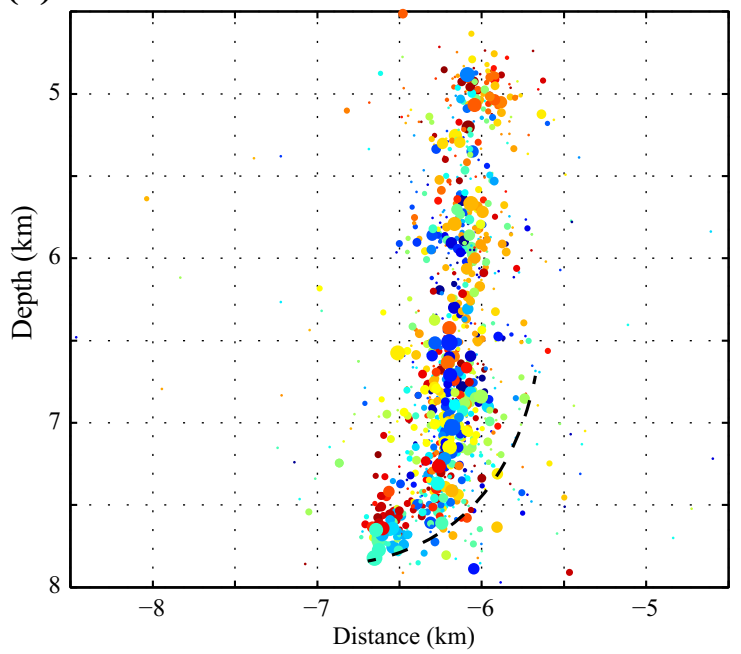

(e)
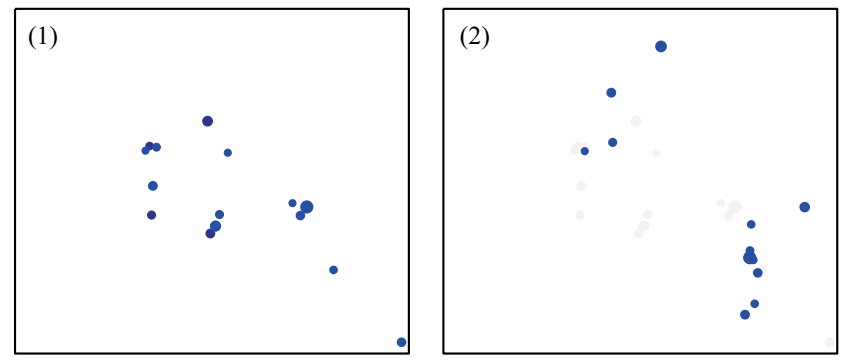

(3)

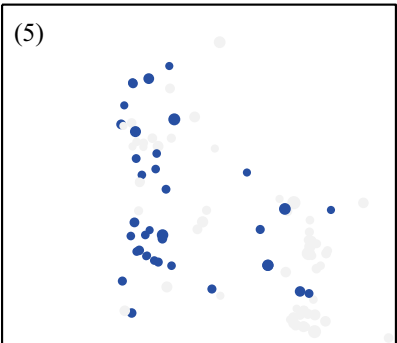

(6)

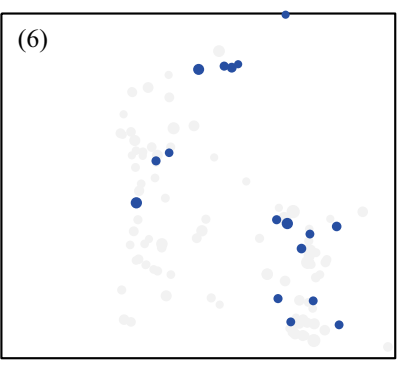

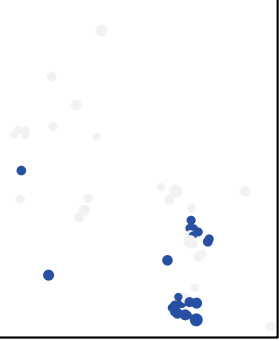

(7)

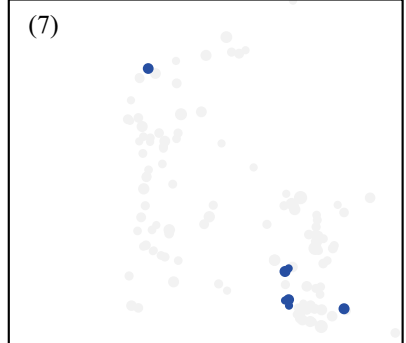

(4)

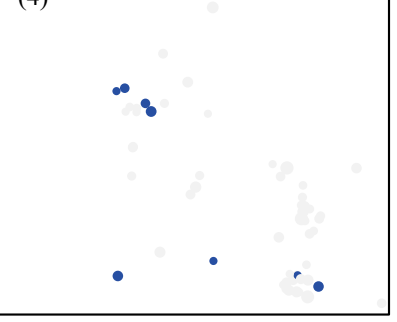

elapsed time from May 6, 2014

(1) 0 -10 days

(2) $10-15$

(3) $15-25$

(4) $25-30$

(5) $30-40$

(6) $40-50$

(7) $50-60$ 
४Fig. 5 Hypocenter distribution data collected from the Rushan earthquake swarm between May 6, 2014, and June 30, 2014. This relocation catalog was generated using temporary network observations and the HypoDD method. a Distribution of aftershock epicenters. The colored scale on this figure denotes the times of events from May 6, 2014, onwards. These data show that the aftershock distribution of the Rushan earthquake swarm conforms to a WNWESE direction. The bold solid green line drawn between points $A$ and $B$ shows the position of a section with approximately corresponds to the fault plane. b Distance to the first event versus time data for relocated hypocenters. In this case, time is counted as that elapsed from the first event in the catalog. c Sources projected onto the fault plane, the initial coordinates is rotated with the strike angle. $\mathbf{d}$ Profiles of hypocenters along the section between $A$ and $B$, also in a rotated coordinates, the black dashed line denotes a supposed fault. e Evolution of swarm activities with $M_{\mathrm{L}} \geq 1.0$ (black dots) in different periods (the range for each small diagram is same with that in panel (c)), while gray dots denote all these $M_{\mathrm{L}} \geq 1.0$ events in relocated catalog. Detailed explanation is provided in Sect. 2.3 above

15 returned to the right-bottom side, while clusters 12 and 13 continued to be active on the right-top side. Although there appears to be no evidence for an overall directional migration of seismicity, the activities of the different clusters nevertheless reveal some order to their occurrences.

\subsection{Hydraulic diffusivity of clusters}

As shown in previous works (Shapiro et al. 1997; Hainzl 2004; Hainzl and Ogata 2005; Hainzl et al. 2012; Shelly et al. 2013a, b), although $D$ is generally expected to range

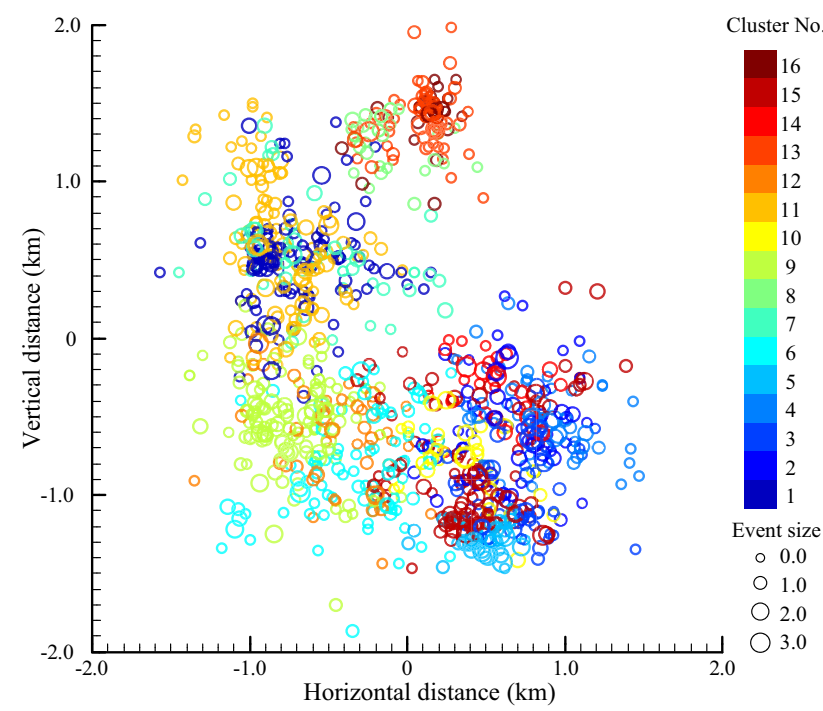

Fig. 6 Cluster analysis results for relocated hypocenters within the Rushan earthquake swarm for the period between May 6, 2014, and June 30, 2014. Hypocenters in the same color belong to the same cluster, and all are projected onto the fault plane (Fig. 5c). The origin of the coordinates used here was defined as the center of all events within the time period between 0.01 and $10 \mathrm{~m}^{2} \mathrm{~s}^{-1}$ in the crust (Scholz 2002), this can also vary depending on crustal medium. One extreme example was presented by Noir et al. (1997) who reported a $D$ value of about $2 \mathrm{~km}^{2} / \mathrm{min}$ for a sequence in Central Afar, and a high diffusion rate also characterizes Yellowstone Lake (Farrell et al. 2010).

The swarm activities can be separated into several clusters in time and space domain. We therefore tested the fluid implication hypothesis for the Rushan earthquake swarm by plotting the distance, $d$, between the first located event and all others within each cluster as a function of time, $t$. Unsurprisingly, data reveal a diffusion pattern within every $d-t$ diagram (Fig. 7), and the data envelope also corresponds to the theoretical curve defined by $\sqrt{4 \pi D t}$ (Shapiro et al. 1997). Thus, fitting results (Fig. 7) reveal $D$ values range between 1.2 and $3.5 \mathrm{~m}^{2} / \mathrm{s}$. This data range is in accordance with the majority of other examples (Shapiro et al. 1997; Hainzl 2004; Hainzl and Ogata 2005; Hainzl et al. 2012; Shelly et al. 2013a, b).

Visually, the curve fits of $d-t$ plots are relative poor in some cases (e.g., clusters 3, 4, 8, 9, 13). In our view, the main reason might be that the clusters are separated mathematically, and the criteria are not calibrated. The spread of small rupture is irregular and, to some extent, randomized in rocks; the cluster analysis can not recreate the rupture process completely and correctly.

When we mark the $D$ values for each cluster on the first event in it (Fig. 7b), it is clear that the hydraulic diffusivities at the edge of the source area are lower than for those in the central area. It is easy to understand combining with Fig. 3: At the start of the Rushan earthquake swarm, ruptures were focused in the central area, but with progress of activity of the swarm, crack activity diffused and rocks in this area became highly crushed. These centrally located rocks subsequently developed higher $D$ values as the result of later shocks.

\section{ETAS modeling}

The ETAS model is a self-exciting point process that describes temporal and spatial clustering within earthquake catalogs (Ogata et al. 1993). This approach has been widely utilized to analyze and describe the spatiotemporal characteristics of regional seismic activities and aftershock sequences (e.g., Jiang et al. 2007; Kumazawa and Ogata 2014). The ETAS model has also been shown to be an appropriate tool to extract primary fluid signals from earthquake swarms (Hainzl and Ogata 2005; Lei et al. 2008, 2013; Lombardi et al. 2010; Jiang et al. 2012; Eto et al. 2013). 

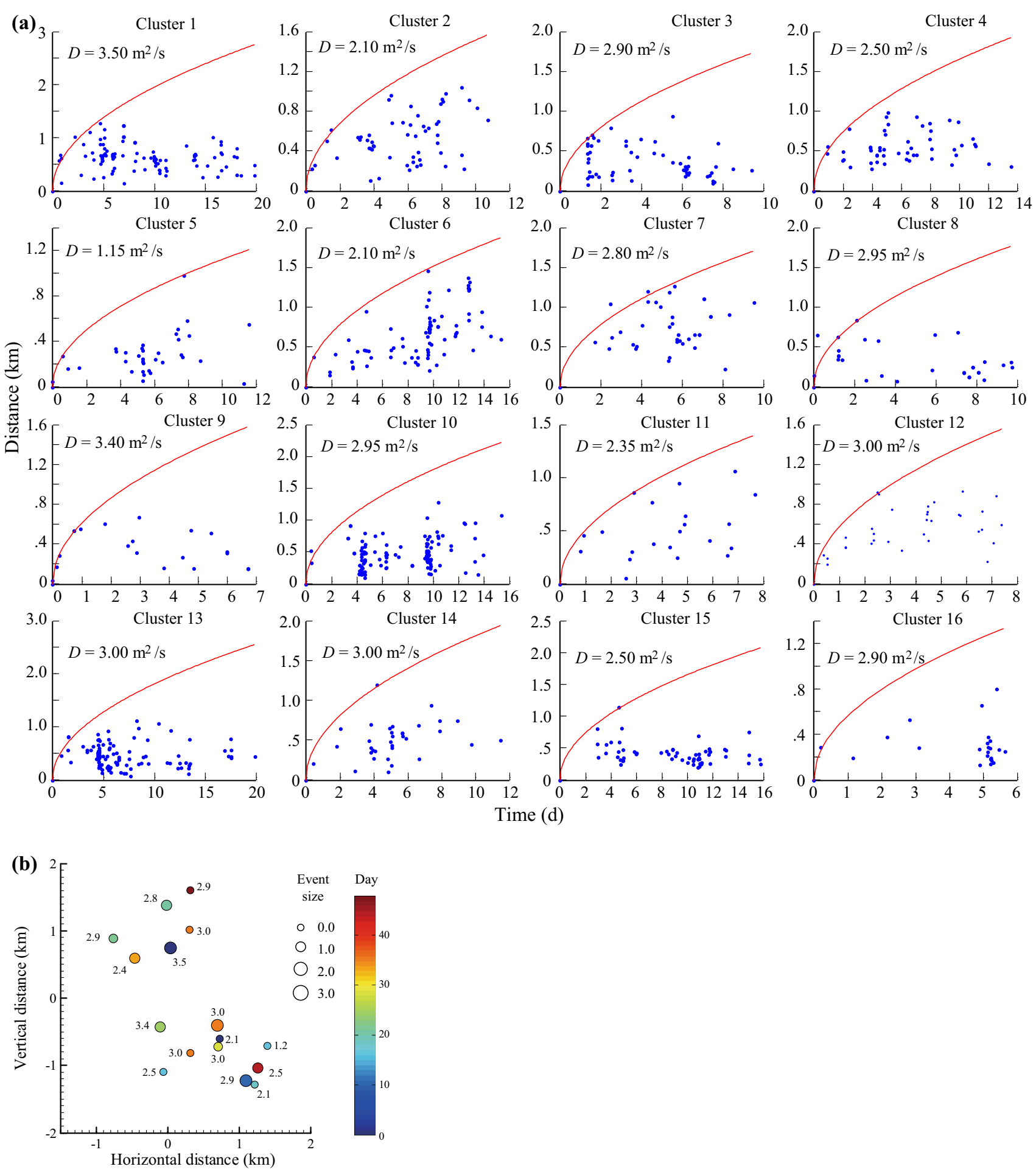

Fig. 7 Data showing the distance between hypocenter and fluid source as a function of earthquake occurrence times for the 16 clusters identified in this study. a Fitted $D$ (red) results for each cluster. b $D$ values for the 16 clusters marked on the first event's hypocenter in each clusters. The circle size is scaled to first event magnitude. Note that the coordinates of this plot are the same as those in Fig. 6 and that events are sorted by time elapsed since May 6, 2014 
In the ETAS model, the rate of aftershock occurrence at time $t$ following the $i$ th earthquake is given as follows:

$n_{i}(t)=K \exp \left[\alpha\left(M_{i}-M z\right)\right] /\left(t-t_{i}+c\right)^{p}$.

In this expression, $p$ means decay rate of aftershocks, $\alpha$ denotes the discrepancy between different events in generating aftershocks, $c$ is relative to degree of seismic activity in the region, and $K$ is equal to the expected number of aftershocks for an event. As this formula describes a self-exciting process which obeys the modified Omori law, the rate of occurrence of the whole earthquake series at time $t$ is as follows:

$\lambda(t)=\mu+\sum_{i} n_{i}(t)$.

In this expression, $\mu$ denotes primary activity at a constant rate of occurrence which can be thought of as background seismicity or activities due to external triggers such as increases in pore pressure (Hainzl and Ogata 2005) or perturbations of stress from large remote earthquakes (Peng et al. 2012). In the case of a fluid-triggered earthquake swarm, $\mu$ has been shown to be consistent with variations in pore pressure and can thus be viewed as a proxy for fluid-driven activity (Hainzl and Ogata 2005; Lei et al. 2008, 2013; Jiang et al. 2012).

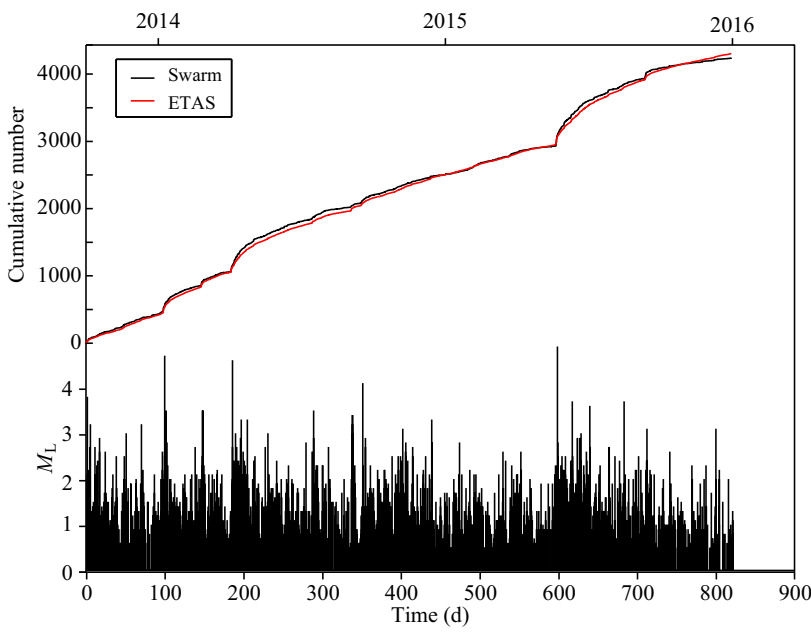

Fig. 8 Estimated ETAS model fit results. The cumulative number of aftershocks is plotted in this figure against ordinary time. The red curve denotes the theoretical cumulative number of detected aftershocks, while the black curve represents the observed cumulative number. An $M-t$ plot of aftershocks is presented beneath the fitted result

\subsection{Estimating ETAS model parameters}

The Rushan region is characterized by low seismic activity. Indeed, as noted above, between January 1, 1970, and September 30, 2013, just 121 weak earthquakes (i.e., $M_{\mathrm{L}}$ 2.0 to $M_{\mathrm{L}} 2.9$ ), 16 medium-sized earthquakes (i.e., $M_{\mathrm{L}} 3.0$ to $M_{\mathrm{L}} 3.9$ ), and three large-scale events (i.e., $M_{\mathrm{L}} 4.0$ to $M_{\mathrm{L}}$ 4.3) are recorded in the CENC catalog within a $50 \mathrm{~km}$ radius of the Rushan earthquake swarm. As the vast majority of these earthquakes are related to the NNE faulting Rushan fault and are extremely rare within the epicenter area of the Rushan earthquake swarm, background seismicity can be ignored for the purposes of this research.

ETAS modeling results for the Rushan earthquake swarm are shown in Fig. 8. The catalog is also due to Shandong local earthquake network, same with Fig. 1. But the data we used in ETAS fitting only contain 4256 events with $M_{\mathrm{L}} \geq M_{\mathrm{C}}$. The observed (black) and predicted (red) cumulative numbers of events show that the fit of the estimated model to known earthquakes is fairly consistent. Estimated ETAS model parameters are listed in Table 1.

The final fitting parameter, $\alpha=1.156$, is equivalent to the resultant value (1.09) estimated by Lei et al. (2008) in their study of earthquake sequences in the Rongchang gas field that were explicitly induced by water injection, as well as the value (1.17) calculated based on microseismicity of the loading stage in the Three Gorges Reservoir (Jiang et al. 2012). At the same time, however, the $\alpha$ value estimated here is slightly lower than those estimated for non-swarm activities (between 1.2 and 3.1), but higher than those calculated for swarm activity (between 0.35 and 0.85) in Japan (Ogata 1992), and for the Vogtland and Western Bohemia swarm (0.73; Hainzl and Ogata 2005). As discussed by Ogata (1992), $\alpha$ is likely to be large if there are few conspicuously large events within the aftershock sequence. This may provide one explanation for the discrepancies in values given by different researchers.

As our fitted ETAS model reveals a low forcing rate $(\mu)$ of ca. 0.15 events per day, a total of about 130 earthquakes are implied throughout the entire period of the swarm period, more than 900 days. This result indicates that about $3 \%$ of all events within the Rushan earthquake swarm were externally triggered, while the remainder are the result of Omori-type self-triggered activity.

Table 1 Estimated ETAS parameters and corresponding AIC (Akaike information criterion) and BIC (Bayesian information criterion) values

\begin{tabular}{llllllll}
\hline Time & $\mu$ & $K$ & $C$ & $\alpha$ & $P$ & AIC & BIC \\
\hline 2013-10-01-2016-12-31 & 0.150 & 5.697 & 0.002 & 1.156 & 1.009 & -10530 & 5260 \\
$2013-10-01-2013-10-30$ & 0.151 & 3.271 & 0.001 & 0.907 & 0.816 & -334.437 & 165.400 \\
\hline
\end{tabular}




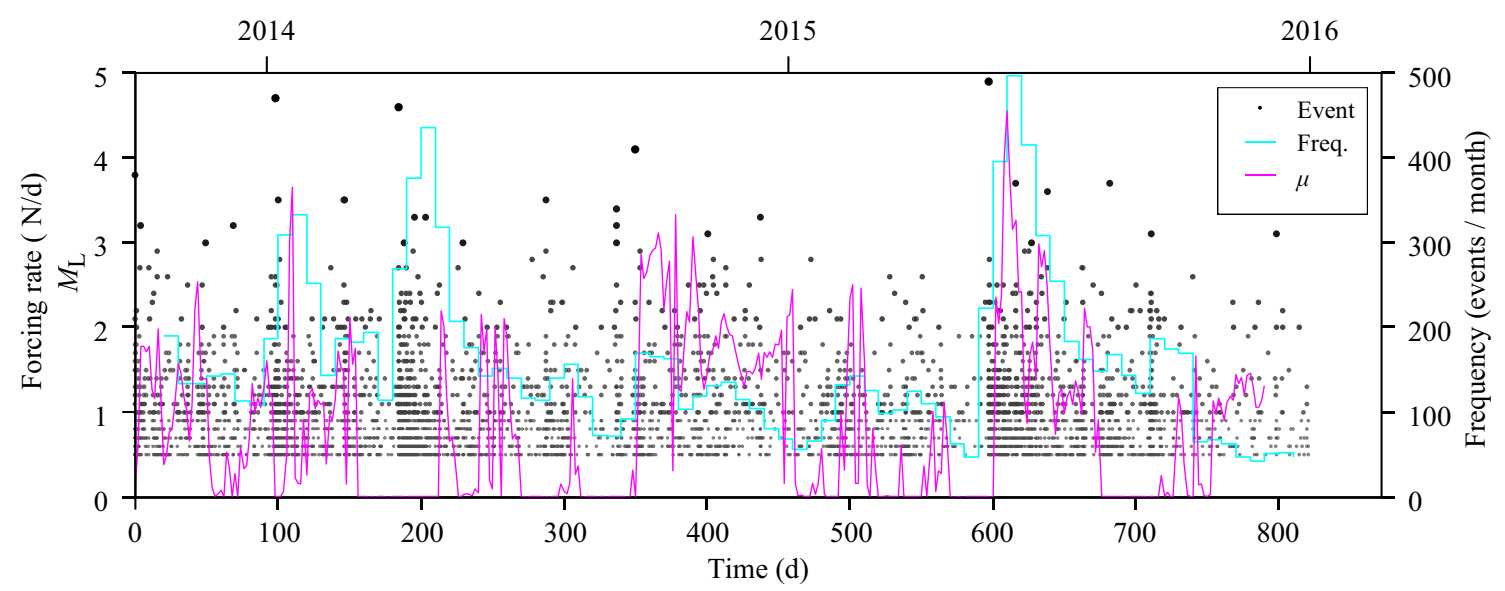

Fig. 9 The relationship between seismic activity and the rate of occurrence of primary fluid signals within the Rushan earthquake swarm. The gray dots in this figure denote the magnitude of all $M_{\mathrm{L}} \geq 0.5$ events, the solid cyan line marks a histogram of monthly swarm frequencies, and the magenta line is the rate of occurrence. Times on the horizontal axis are counted from October 1, 2013

\subsection{Variation of forcing rate $\mu$}

We adapted the method proposed by Hainzl and Ogata (2005) to extract the forcing rate $\mu$, estimating the ETAS parameter using a moving time window with size fixed at 30 days and the moving step set as one day. Although all five parameters were fitted in each time window, initial values were set to those determined for the whole sequence (Table 1). We obtained variation in the occurrence rate of interpreted primary fluid signals, $\mu$, as a function of time elapsed within the Rushan earthquake swarm (Fig. 9). In order to investigate the correlation between $\mu$ and swarm seismicity, the variation in monthly frequency with time was also calculated (Fig. 9).

Results show that variation in $\mu$ is roughly coincident with swarm activity; thus, when $\mu$ is high, aftershocks were active and intense. In other words, a high proportion of fluid-induced events usually occurred subsequent to significant earthquakes. We therefore infer that as the source area became even more crushed by large ruptures due to major events, new fractures emerged and caused the development of additional fluid-triggered cracks.

\section{Discussion and conclusions}

Fluid-driven earthquake swarms have been studied from a number of perspectives (Legrand et al. 2011; Horálek and Š́lený 2013; Leclère et al. 2013; Braunmiller et al. 2014; D'Hour et al. 2016; Schultz et al. 2015). The prevailing consensus is that such swarms are triggered by the rupture of a zone containing confined high-pressure aqueous fluid into a preexisting crustal fault system, which prompts release of accumulated stress (Shelly et al. 2013a, b). However, no such outcropped fault has been located to date within the area of the Rushan earthquake swarm, even during detailed prospecting investigations for gold (Fig. 4; $\mathrm{Hu}$ et al. 2013; Zheng et al. 2015b). In addition, and even more perplexing, as this earthquake swarm is located more than $10 \mathrm{~km}$ away from the nearest fault traces, we have suggested that it might occur on a blind fault $(\mathrm{Qu}$ et al. 2015; Zheng et al. 2015b). The Rushan earthquake swarm is tectonically located at the boundary between two rock mass units (Zheng et al. 2015b); we therefore suggest that, for some reason, the boundary between these two masses was broken and a new fault developed. Cluster analysis of $D$ combined with ETAS modeling further suggests that the reason underlying the development of the Rushan earthquake swarm is deep fluid action.

A relocated catalog for the Rushan earthquake swarm generated using data from a temporary seismic network and the hypoDD method reveals the presence of at least 16 activity clusters between May 6, 2014, and June 30, 2014. The data also show that each of these clusters was itself characterized by a distinct hypocenter spreading process and that the $D$ value of each ranged between 1.2 and $3.5 \mathrm{~m}^{2} / \mathrm{d}$. Results also show lower $D$ values at the margins of the source area than in the center, which may also imply differences in the degrees to which rocks were crushed.

Sibson (1996) studied the structural permeability of fluid-driven fault fracturing, while Yamashita (1999) modeled the spatiotemporal variation in rupture activity assuming fluid migration along a narrow and porous fault zone. The results of both these studies showed that when an inhomogeneity is introduced into a permeability spatial distribution, high complexity rupture activity can result, both spatially and temporally. The multi-cluster activity of the Rushan earthquake swarm reported in this study is in accordance with this earlier work. 
We have estimated the parameters of the Rushan earthquake swarm using ETAS modeling. The final fitting parameter $(\alpha=1.156)$ reported in this paper is equivalent to those previously calculated for earthquake sequences induced by water injection. Calculated $\mu$ for the Rushan swarm (just 0.15 events per day) also indicates that around $3 \%$ of events were fluid-triggered. Finally, the variation in $\mu$ we report here is approximately coincident with earthquake swarm activity, which might imply that the Rushan event was fluid-driven. Taken in combination with our cluster analysis, the migration of underground fluid likely exerted a marked influence on swarm activity.

Acknowledgements The software package of ASAeis2006 written by Prof. Yosihito Ogata is used to perform ETAS analysis. We wish to thank Dr. Haikun Jiang (CENC) for constructive advice on ETAS modeling, as well as Dr. Junhao Qu, Xiaoyi Fan, Juan Mu, Po Li, Yajun Li, and Xiucai Wei for their hard work in phase picking. This study was supported financially by the Science and Technology Development Plan Project of Shandong Province (2014GSF120007), and Shandong Earthquake Agency, China Earthquake Administration (SD1250501). We are grateful to two anonymous reviewers for their helpful comments and suggestions.

Open Access This article is distributed under the terms of the Creative Commons Attribution 4.0 International License (http://crea tivecommons.org/licenses/by/4.0/), which permits unrestricted use, distribution, and reproduction in any medium, provided you give appropriate credit to the original author(s) and the source, provide a link to the Creative Commons license, and indicate if changes were made.

\section{References}

Bourouis S, Cornet FH (2009) Microseismic activity and fluid fault interactions: some results from the Corinth Rift Laboratory (CRL), Greece. Geophys J Int 178(1):561-580

Braunmiller J, Nábělek JL, Tréhu AM (2014) A seasonally modulated earthquake swarm near Maupin, Oregon. Geophys J Int 197(3):1736-1743

Cappa F, Rutqvist J, Yamamoto K (2009) Modeling crustal deformation and rupture processes related to upwelling of deep $\mathrm{CO}_{2}$ rich fluids during the 1965-1967 Matsushiro earthquake swarm in Japan. J Geophys Res 114:B10304

Cheng Y (1995) Mean shift, mode seeking, and clustering. IEEE Trans Pattern Anal Mach Intell 17(8):790-799

Comaniciu D, Meer P (2002) Mean shift: a robust approach toward feature space analysis. IEEE Trans Pattern Anal Mach Intell 24(5):603-619

Davis SD, Frohlich C (1991) Single-link cluster analysis of earthquake aftershocks: decay lays and regional variations. J Geophys Res 96(B4):6335-6350

D'Hour V, Schimmel M, Do Nascimento AF, Ferreira JM, Lima Neto HC (2016) Detection of subtle hydromechanical medium changes caused by a small-magnitude earthquake swarm in NE Brazil. Pure Appl Geophys 173(4):1097-1113

Dzwinel W, Yuen DA, Boryczko K, Ben-Zion Y, Yoshioka S, Ito T (2005) Cluster analysis, data-mining, multi-dimensional visualization of earthquakes over space, time and feature space. Seismol Res Lett 12:117-128
Eto T, Asanuma H, Adachi M, Saeki K, Aoyama K, Ozeki H, Mukuhira Y, Haring M (2013) Application of the ETAS seismostatistical model to microseismicity from geothermal fields. GRC Trans 37:149-154

Farrell J, Smith RB, Taira TA, Chang WL, Puskas CM (2010) Dynamics and rapid migration of the energetic 2008-2009 Yellowstone Lake earthquake swarm. Geophys Res Lett 37(19):L19305

Frohlich C, Davis SD (1990) Single-link cluster analysis as a method to evaluate spatial and temporal properties of earthquake catalogues. Geophys J Int 100(1):19-32

Godano M, Larroque C, Bertrand E, Courboulex F, Deschamps A, Salichon J, Blaud- Guerry C, Fourteau L, Charlety J, Deshayes P (2013) The October-November 2010 earthquake swarm near Sampeyre (Piedmont region, Italy): a complex multicluster sequence. Tectonophysics 608:97-111

Grünthal G, Schenk V, Zeman A, Schenková Z (1990) Seismotectonic model for the earthquake swarm of 1985-1986 in the Vogtland/West Bohemia focal area. Tectonophysics 174(3-4):369-383

Hainzl S (2004) Seismicity patterns of earthquake swarms due to fluid intrusion and stress triggering. Geophys J Int 159(3):1090-1096

Hainzl S, Fischer T (2002) Indications for a successively triggered rupture growth underlying the 2000 earthquake swarm in Vogtland/NW Bohemia. J Geophys Res Solid Earth 107(B12):2338

Hainzl S, Ogata Y (2005) Detecting fluid signals in seismicity data through statistical earthquake modeling. J Geophys Res Solid Earth 110(B05):S07

Hainzl S, Fischer T, Dahm T (2012) Seismicity-based estimation of the driving fluid pressure in the case of swarm activity in Western Bohemia. Geophys J Int 191(1):271-281

Hauksson E, Stock J, Bilham R, Boese M, Chen X, Fielding EJ (2013) Report on the August 2012 Brawley earthquake swarm in Imperial Valley, southern California. Seismol Res Lett 84(2):177-189

Holland PW, Welsch RE (1977) Robust regression using iteratively re-weighted least-squares. Commun Stat Theory Methods A6(1977):813-827

Horálek J, Šílený J (2013) Source mechanisms of the 2000 earthquake swarm in the West Bohemia/Vogtland region (Central Europe). Geophys J Int 194:979-999

Hu F, Fan H, Yang J, Wan Y, Liu D, Zhai M, Jin C (2013) Mineralizing age of the Rushan lode gold deposit in the Jiaodong Peninsula: SHRIMP U-Pb dating on hydrothermal zircon. Chin Sci Bull 49(15):1629-1636

Jenatton L, Guiguet R, Thouvenot F, Daix N (2007) The 16,000-event 2003-2004 earthquake swarm in Ubaye (French Alps). J Geophys Res Solid Earth 112:B11304

Jiang HK, Zheng JC, Wu Q, Qu YJ, Li YL (2007) Earlier statistical features of ETAS model parameters and their seismological meanings. Chin J Geophys 50(6):1778-1786 (in Chinese with English abstract)

Jiang HK, Song J, Wu Q, Li J, Qu JH (2012) Quantitative investigation of fluid triggering on seismicity in the Three Geoges Reservior area base on ETAS model. Chin J Geophys 55(7):2341-2352 (in Chinese with English abstract)

Kishimoto Y, Hasshizume M, Oike K, Mino K, Kurita T, Nishida R, Watanabe K, Matsuo S (1967) Seismometric observations of Matsuchiro swarm. Bull Disaster Prev Res Inst Kyoto Univ 17(1):9-26

Kumazawa T, Ogata Y (2014) Nonstationary ETAS models for nonstandard earthquakes. Ann Appl Stat 8(3):1825-1852

Leclere H, Daniel G, Fabbri O, Cappa F, Thouvenot F (2013) Tracking fluid pressure buildup from focal mechanisms during 
the 2003-2004 Ubaye seismic swarm, France. J Geophys Res Solid Earth 118(8):4461-4476

Legrand D, Barrientos S, Bataille K, Cembrano J, Pavez A (2011) The fluid-driven tectonic swarm of Aysen Fjord, Chile (2007) associated with two earthquakes $\left(M_{\mathrm{W}}=6.1\right.$ and $\left.M_{\mathrm{W}}=6.2\right)$ within the Liquine-Ofqui fault zone. Cont Shelf Res 31(3-4):154-161

Lei X, Yu G, Ma S, Wen X, Wang Q (2008) Earthquakes induced by water injection at $3 \mathrm{~km}$ depth within the Rongchang gas field, Chongqing, China. J Geophys Res 113:B10310

Lei X, Ma S, Chen W, Pang C, Zeng J, Jiang B (2013) A detailed view of the injection-induced seismicity in a natural gas reservoir in Zigong, southwestern Sichuan Basin, China. J Geophys Res 118(8):4296-4311

Lindenfeld M, Rumpker G, Link K, Koehn D, Batte A (2012) Fluidtriggered earthquake swarms in the Rwenzori region, East African Rift-evidence for rift initiation. Tectonophysics 566-567:95-104

Liu XL, Liu TT, Zheng JC, We YH, Ma YX (2007) Study on the characteristics of small seismic swarms occurring in Shandong Province and its nearby ocean floor. J Disaster Prev Mitig Eng 27(4):457-464 (in Chinese)

Lombardi MA, Cocco M, Marzocchi W (2010) On the increase of background seismicity rate during the 1997-1998 UmbriaMarche, Central Italy, sequence: apparent variation or fluiddriven triggering? Bull Seismol Soc Am 100(3):1138-1152

Noir J, Jacques E, Bekri S, Adler PM, Tapponnier P, King GCP (1997) Fluid flow triggered migration of events in the 1989 Dobi earthquake sequence of Central Afar. Geophys Res Lett 24(18):2335-2338

Ogata Y (1992) Detection of precursory relative quiescence before great earthquakes through a statistical model. J Geophys Res 97(B13):19845-19871

Ogata Y, Matsu'ura RS, Katsura K (1993) Fast likelihood computation of epidemic type aftershock sequence model. Geophys Res Lett 20:2143-2146

Peng Y, Zhou S, Zhuang J, Shi J (2012) An approach to detect the abnormal seismicity increase in Southwestern China triggered co-seismically by 2004 Sumatra $M_{\mathrm{W}} 9.2$ earthquake. Geophys J Int 189:1734-1740

Qu JH, Jiang HK, Li J, Zhang ZH, Zheng JC, Zhang Q (2015) Preliminary study for seismogenic structure of the Rushan earthquake sequence in 2013-2014. Chin J Geophys 58(6):1954-1962 (in Chinese with English abstract)

Rehman K, Burton PW, Weatherill GA (2014) K-means cluster analysis and seismicity partitioning for Pakistan. J Seismol 18(3):401-419

Scholz CH (2002) The mechanics of earthquakes and faulting. Cambridge University Press

Schultz R, Mei S, Pană D, Stern V, Gu YJ, Kim A, Eaton D (2015) The Cardston earthquake swarm and hydraulic fracturing of the Exshaw formation (Alberta Bakken play). Bull Seismol Soc Am 105(6):2871-2884

Shapiro SA, Huenges E, Borm G (1997) Estimating the crust permeability from fluid-injection-induced seismic emission at the KTB site. Geophys J Int 131(2):F15-F18

Shearer P, Hauksson E, Lin G (2005) Southern California hypocenter relocation with waveform cross-correlation, part 2: results using source-specific station terms and cluster analysis. Bull Seismol Soc Am 95(3):904-915

Shelly DR, Hill DP, Massin F, Farrell J, Smith RB, Taira T (2013a) A fluid-driven earthquake swarm on the margin of the Yellowstone caldera. J Geophys Res Solid Earth 118:4872-4886

Shelly DR, Moran SC, Thelen WA (2013b) Evidence for fluidtriggered slip in the 2009 Mount Rainier, Washington earthquake swarm. Geophys Res Lett 40(8):1506-1512
Sibson RH (1996) Structural permeability of fluid-driven faultfracture meshes. J Struct Geol 18(8):1031-1042

Stuart WD, Johnston MJS (1975) Intrusive origin of the Matsushiro earthquake swarm. Geology 3(2):63-67

Teanby NA, Kendall JM, van der Baan M (2004) Automation of shear-wave splitting measurements using cluster analysis. Bull Seismol Soc Am 94(2):453-463

Waldhauser F, Ellsworth WL (2000) A double-difference earthquake location algorithm: method and application to the Northern Hayward Fault, California. Bull Seismol Soc Am 90(6):1353-1368

Wang P, Zheng JC (2014) Temporal and spatial variation of apparent stress in Eastern Shandong Province. Earthquake 34(4):70-77 (in Chinese with English abstract)

Weatherill G, Burton PW (2009) Delineation of shallow seismic source zones using $K$-means cluster analysis, with application to the Aegean region. Geophys J Int 176(2):565-588

Wei S, Helmberger D, Owen S, Graves RW, Hudnut KW, Fielding EJ (2013) Complementary slip distributions of the largest earthquakes in the 2012 Brawley swarm, Imperial Valley, California. Geophys Res Lett 40(5):847-852

Wei S, Avouac JP, Hudnut KW, Donnellan A, Parker JW, Graves RW, Helmberger D, Fielding E, Liu Z, Cappa F, Eneva M (2015) The 2012 Brawley swarm triggered by injection-induced aseismic slip. Earth Planet Sci Lett 422:115-125

Yamashita T (1997) Mechanical effect of fluid migration on the complexity of seismicity. J Geophys Res Solid Earth 102(B8):17797-17806

Yamashita T (1999) Pore creation due to fault slip in a fluidpermeated fault zone and its effect on seismicity: generation mechanism of earthquake swarm. Pure Appl Geophys 155:625-647

Yang M, Qu Y (1999) Robust estimation of $b$ value and its application to earthquake prediction. Earthquake 19(3):253-260 (in Chinese with English abstract)

Zaliapin I, Ben-Zion Y (2011) Asymmetric distribution of aftershocks on large faults in California. Geophys J Int 185(3):1288-1304

Zaliapin I, Ben-Zion Y (2013a) Earthquake clusters in southern California I: identification and stability. J Geophys Res 118(6):2847-2864

Zaliapin I, Ben-Zion Y (2013b) Earthquake clusters in southern California II: classification and relation to physical properties of the crust. J Geophys Res 118(6):2865-2877

Zaliapin I, Gabrielov A, Keilis-Borok V, Wong H (2008) Clustering analysis of seismicity and aftershock identification. Phys Rev Lett 101(018501):1-4

Zhang HF, Li SR, Zhai MG, Guo JH (2006) Crust uplift and its implications in the Jiaodong Peninsula, eastern China. Acta Petrol Sin 22(2):285-295

Zheng JC, Zhang YX, Pan YS, Wu YJ, Wan LC (2006) Analysis on variation of ambient shear stress and apparent stress at Laoshan, Qingdao area. Earthquake 26(3):123-130 (in Chinese with English abstract)

Zheng JC, Li DM, Wang P, Lv ZQ, Lin M (2014) Method and application of clustering seismicity identification base on nearest-neighbor distance. Earthquake 34(4):100-109 (in Chinese with English abstract)

Zheng JC, Lin M, Wang P, Xu CP (2015a) Error analysis for focal mechanisms from CAP method inversion: an example of 2 moderate earthquakes in Jiaodong Peninsula. Chin J Geophys 58(2):453-462 (in Chinese with English abstract)

Zheng JC, Qu L, Qu JH, Hu XH, Li DM (2015b) Comparison of locations of Rushan earthquake swarm from large and small network. Seismol Geol 37(4):953-965 (in Chinese with English abstract) 